\title{
TRANSTORNOS DEPRESSIVOS ASSOCIADOS À NEUROCISTICERCOSE PREVALÊNCIA E CORRELAÇÕES CLÍNICAS
}

\author{
ORESTES VICENTE FORLENZA*, ANTÔNIO H. GUERRA VIEIRA FILHO* \\ LUIS DOS RAMOS MACHADO**, JOSÉ PAULO S. NÓBREGA **, NÉLIO GARCIA DE BARROS ***
}

\begin{abstract}
RESUMO - Objetivo: Investigar a ocorrência e os fatores de risco para morbidade depressiva em uma amostra de 38 pacientes ambulatoriais com neurocisticercose. Métodos: O diagnóstico psiquiátrico baseou-se nos exames do estado mental atual e pregresso, respectivamente obtidos pelas entrevistas estruturadas do PSE-9 e SADS-L; as funções cognitivas foram avaliadas pelo MMS e pelo roteiro de Strub \& Black (Mental Status Examination). Resultados: Transtornos depressivos foram o achado psiquiátrico mais frequente $(63,1 \%)$ entre os pacientes da amostra. Destes, 20 (52,6\%) mostravam-se deprimidos no momento da avaliação e 4 apresentaram depressão no passado. Entre os pacientes deprimidos, 16 preencheram critérios diagnósticos do DSM-III-R para transtorno orgânico do humor $(k=0,4)$. Antecedentes pessoais de depressão $(\mathrm{p}=0,006)$, sinais de atividade da doença $(\mathrm{p}=$ $0,044)$ e ocorrência de hipertensão intracraniana $(\mathrm{p}=0,065)$ foram os parâmetros clínicos que se correlacionaram com a presença de depressão. Conclusões: Tais achados, aliados à ausência de predomínio do sexo feminino entre os casos de depressão, sugerem etiologia orgânica. Os autores discutem essas observações à luz da literatura sobre outros transtornos mentais orgânicos.
\end{abstract}

PALAVRAS-CHAVE: neurocisticercose, transtornos (distúrbios) mentais orgânicos, depressão.

\section{Depressive disorders associated with neurocysticercosis: prevalence and clinical correlations}

ABSTRACT - Objective: To determine the frequency and features of psychiatric morbidity in a cross-section of 38 outpatients with neurocysticercosis. Methods: Diagnosis of neurocysticercosis was established by CT scan, MRI and CSF analysis. Psychiatric diagnoses were made by using the Present State Examination and the Schedule for Affective Disorders and Schizophrenia - Lifetime version; cognitive state was assessed by Mini-Mental State Examination and Strub \& Black's Mental Status Examination. Results: Depression was the most frequent psychiatric diagnosis $(52.6 \%)$ as shown by PSE. Active disease and intracranial hypertension were associated with higher psychiatric morbidity, and previous history of mood disorders was strongly related to current depression. Conclusions: Depression syndromes are frequent in patients with neurocysticercosis. The extent to which organic mechanisms related to brain lesions may underlie the observed mental changes is yet unclear, though the similar sex distribution of patients with and without depression, as well as the above mentioned correlations, provide further evidence of the role played by organic factors in the cause of these syndromes. The results of this study are discussed in the light of the data available for other organic psychiatric disorders.

KEY WORDS: neurocysticercosis, organic mental disorders, depression.

A neurocisticercose (NCC) é a mais comum das parasitoses do sistema nervoso central (SNC) humano ${ }^{1}$ e corresponde à infestação dele pela forma larvária do cestóide Taenia solium. É doença própria dos países ou regiões onde há contaminação dos rebanhos suínos, consumo de carne suína

* Laboratório de Investigações Médicas (LIM-23) do Hospital das Clínicas da Faculdade de Medicina da Universidade de São Paulo (HC/FMUSP) e Instituto de Psiquiatria do HC/FMUSP; ** Divisão de Clínica Neurólogica do HC/FMUSP; *** Serviço de Neuro-Radiologia do HC/FMUSP. Aceite: 22-novembro-1997.

Dr. Orestes Vicente Forlenza - Instituto de Psiquiatria, Hospital das Clínicas, FMUSP - Rua Ovídio Pires de Campos s/n - 05403-010 São Paulo SP - Brasil. 
(parasitada) e condições de saneamento básico e hábitos de higiene deficientes. Ocorre endemicamente em diversos países da Ásia, África e América Latina, destacando-se México e Brasil (região Centro$\mathrm{Sul}$ ). A prevalência da NCC em áreas endêmicas varia entre 0,1 e 3,6\% ${ }^{2-9}$. Oriunda do meio rural, tem sido observada com frequência crescente também em zonas urbanas. Acomete predominantemente indivíduos com idades entre 21 e 40 anos, com grande morbidade clínica e psiquiátrica ${ }^{10-13}$.

A Taenia solium é um parasita hermafrodita do intestino delgado. Seu ciclo biológico envolve o homem, único hospedeiro definitivo da tênia adulta, e o porco, hospedeiro intermediário da forma larvária do cestóide, o cisticerco. $\mathrm{O}$ homem usualmente adquire a cisticercose quando ingere água ou alimentos contaminados com os ovos do parasita, eliminados através das fezes do portador do verme adulto ${ }^{14,15}$. O Cysticercus cellulosae tem tropismo pelos tecidos subcutâneo, muscular, ocular e cerebral, onde evoluem para forma cística, permanecendo quiescentes por vários meses ou anos ${ }^{9}$. A degeneração dos cistos (espontânea ou induzida por drogas parasiticidas) é acompanhada de processo inflamatório, fibrose e calcificação. A agressão ao SNC dá-se por três processos: pela presença do parasita no parênquima cerebral ou nos espaços liquóricos; pelo processo inflamatório decorrente; ou pela formação de fibroses, granulomas e calcificações residuais. A patologia cerebral depende do número, tipo e localização dos cistos, além da resposta imunológica do hospedeiro ${ }^{16-18}$. A presença de cisticercos no espaço liquórico pode determinar obstrução do fluxo líquido cefalorraquidiano (LCR), hipertensão intracraniana (HIC) e hidrocefalia. Cisticercos parenquimatosos podem dar origem a processos compressivos, irritativos, vasculares e obstrutivos. Embora haja casos assintomáticos, sua degeneração correlaciona-se com o início das manifestações clínicas, em função do edema dos tecidos adjacentes e da ruptura da barreira hemato-encefálica (BHE), surgindo reações locais e à distância. As calcificações correspondem à forma "cicatricial" da NCC, estando associada a epileptogênese, espessamentos meníngeos e alterações do fluxo do LCR. As manifestações clínicas mais comuns são as síndromes epilépticas, hipertensivas, meningíticas e focais. A NCC é a principal causa da epilepsia tardia em áreas endêmicas. Alterações mentais são frequentes no decurso da doença, tendo sido descritas síndromes esquizofreniformes, transtornos do humor e deterioração cognitiva ${ }^{19}$.

Este estudo tem como objetivos: 1. descrever e estimar a prevalência de transtornos mentais, particularmente do humor, em amostra de pacientes com NCC do Ambulatório de Moléstias Neuroinfecciosas da Clínica Neurológica do Hospital das Clínicas da FMUSP; 2. investigar os possíveis fatores de risco para o aumento da morbidade psiquiátrica nos pacientes acometidos, confrontando os achados com a literatura sobre doença mental orgânica.

\section{CASUÍSTICA E MÉTODOS}

\section{Pacientes}

Foram incluídos no estudo 38 pacientes portadores de NCC, com idades entre 18 e 60 anos, após consentimento informado. Os pacientes foram incluídos de forma sequencial e não-selecionada, correspondendo a todos os casos recém-admitidos no serviço. O diagnóstico de NCC provável foi estabelecido por critérios clínico-neurológicos, exame do $\mathrm{LCR}^{20-22}$ e tomografia computadorizada de crânio (TC) ${ }^{23}$. Imagens por ressonância magnética do encéfalo (RM) foram obtidas com o intuito de aumentar a sensibilidade diagnóstica e para a detecção de edema perilesional ${ }^{24}$.

Foram excluídos da amostra pacientes portadores de doenças clínicas ou neurológicas concomitantes, não relacionadas à NCC, que pudessem cursar com manifestações psiquiátricas. Usuários de álcool ou substâncias psicoativas, salvo aquelas necessárias para o tratamento da NCC, também foram excluídos do estudo.

\section{Procedimentos}

Os pacientes selecionados para o estudo foram submetidos a avaliação clínica (coleta sistematizada de dados clínico-demográficos), laboratorial (exame químio-citológico do LCR e provas imunológicas) e radiológica (contagem, caracterização e localização das lesões cerebrais identificada à TC e RM). A avaliação psiquiátrica baseou-se nos escores obtidos pelos seguintes intrumentos de avaliação:

1. "Schedule for Affective Disorders and Schizophrenia-lifetime version"(SADS-L): entrevista estruturada para avaliação da história psiquiátrica pregressa ${ }^{25}$. 
2. "Present State Examination" (PSE): entrevista estruturada para caracterização do estado mental atual ${ }^{26}$.

3. Critérios diagnósticos do DSM-III-R ${ }^{27}$, aplicados aos pacientes com possível doença mental segundo as entrevistas acima.

4. Mini-exame do Estado Mental (MMSE): avaliação cognitiva sumária ${ }^{28}$, utilizando-se pontos de corte diferenciados segundo o nível de instrução (13 para analfabetos, 18 para indivíduos com até 8 anos de escolaridade e 26 para aqueles com mais de 8$)^{29}$.

5. Mental Status Examination (MSE): avaliação detalhada da atenção, memória, linguagem (leitura e escrita), habilidades visoespaciais, funções executivas (praxia e funções motoras) e funções cognitivas superiores ${ }^{30}$.

\section{Classificação}

Os casos foram classificados segundo a localização das lesões cerebrais (NCC parenquimatosa, cisternoventricular, sub-aracnóide e mista) e sinais de atividade da doença ${ }^{17}$. Foram considerados portadores de NCC inativa os pacientes com lesões parenquimatosas calcificadas e LCR sem sinais de quebra da BHE; NCC ativa foi caracterizada pela presença de cistos íntegros ou em degeneração, ou LCR inflamatório (i.e., com pleocitose e hiperproteinorraquia).

\section{RESULTADOS}

As variáveis demográficas e clínicas encontram-se na Tabela 1. Sinais de atividade da doença foram observados em 29 casos (76,3\%), 11 dos quais vinham fazendo uso de corticosteróides no momento das avaliações $(28,9 \%)$. As lesões parenquimatosas eram geralmente múltiplas e disseminadas, em diferentes estágios evolutivos. Apenas 5 pacientes apresentavam cisto único; 15

Tabela 1. Variáveis demográficas $(n=38)$ e clínico-laboratoriais.

$\begin{array}{llll}\text { Idade } & \text { anos (média): } & 36,7 & (18-59) \\ \text { Sexo } & \text { homens } & 18 & (47,4 \%) \\ \text { mulheres } & 20 & (52,6 \%) \\ \text { Raça } & \text { brancos } & 27 & (71,0 \%) \\ & \text { negros } & 3 & (7,9 \%) \\ \text { mestiços } & 8 & (21,1 \%) \\ \text { Nível de instrução } & \text { analfabetos } & 3 & (7,9 \%) \\ & \text { primeiro grau } & 22 & (57,9 \%) \\ & \text { segundo grau } & 10 & (26,3 \%) \\ \text { nivel superior } & 3 & (7,9 \%) \\ \text { Estado civil } & \text { casados } & 22 & (57,9 \%) \\ & \text { solteiros } & 13 & (34,2 \%) \\ \text { Manifestação inicial } & \text { outros } & 3 & (7,9 \%) \\ \text { (n=38) } & \text { psiquiátrica } & 3 & (7,9 \%) \\ & \text { neurológica } & 31 & (81,6 \%) \\ \text { Síndrome clínica } & \text { assintomáticos } & 4 & (10,5 \%) \\ (\mathrm{n}=34) & \text { epiléptica (E) } & 23 & (67,6 \%) \\ & \text { hipertensiva }(\mathrm{H}) & 3 & (8,8 \%) \\ & \text { mista (E + H) } & 3 & (8,8 \%) \\ \text { Localização das lesões } & \text { outras } & 5 & (14,7 \%) \\ (\mathrm{n}=38) & \text { parenquimatosa } & 20 & (52,6 \%) \\ & \text { espaço do LCR } & 7 & (18,4 \%) \\ \text { LCR } & \text { meníngea } & 0 & (0 \%) \\ & \text { mista } & 11 & (28,9 \%) \\ & \text { sem alterações } & 20 & (54,1 \%) \\ & \text { atividade inflamatória } & 6 & (16,2 \%) \\ & \text { atividade inflamatória }+ & & (29,7 \%) \\ & \text { reações imunológicas positivas } & 11 & \end{array}$


Tabela 2. Distribuição das lesões parenquimatosas $(n=31)$ segundo as regiões cerebrais acometidas: número de pacientes com lesões nas respectivas localizações.

\begin{tabular}{ccc}
\hline & Hemisfério direito & Hemisfério esquerdo \\
\hline Lobo frontal & 15 & 15 \\
Lobo temporal & 15 & 13 \\
Lobo parietal & 16 & 23 \\
Lobo occipital & 15 & 15 \\
Núcleos da base & 11 & 9 \\
Tálamo & 7 & 11 \\
\hline
\end{tabular}

tinham 2 a 5 lesões, e 6, mais de 20 lesões (um dos quais com mais de 300, entre cistos e calcificações). Não se identificou um padrão localizatório para a distribuição dos cistos parenquimatosos. Ambos os hemisférios encontravam-se igualmente acometidos, com lesões em todas as áreas corticais e estruturas subcorticais (tálamo e gânglios da base) (Tabela 2). Cistos racemosos foram identificados em 3 casos.

O diagnóstico psiquiátrico baseou-se em evidências pregressas e atuais de doença mental. Quinze pacientes não tinham antecedentes psiquiátricos e 23 (60,5\%) apresentavam sinais de doença mental prévia, segundo o SADS-L. Entre estes, 42 diagnósticos foram estabelecidos, sugerindo que alguns pacientes receberam mais de um diagnóstico ao longo da vida (Tabela 3). Transtornos depressivos (depressão maior, menor e intermitente) foram observados em 15 pacientes ( 3 dos quais com mais de um subtipo diagnosticado).

Treze pacientes $(34,2 \%)$ foram considerados mentalmente sadios pelo PSE (índice de definição <5), enquanto $25(65,8 \%)$ apresentavam manifestações psiquiátricas leves ou moderadas.

Tabela 3. Frequência de diagnósticos SADS-L/RDC (Research Diagnostic Criteria) em 23 casos NCC.

\begin{tabular}{lccc}
\hline \multicolumn{1}{c}{ SADS-L / RDC } & $n$ & $(\%)$ & Total (3 classes) \\
\hline Depressão maior & $\mathbf{1 2}$ & $\mathbf{( 5 2 , 2 )}$ & $\mathbf{1 8 ~ / 3 8 ~ ( 4 7 , 3 \% )}$ \\
Depressão menor & $\mathbf{1}$ & $\mathbf{( 4 , 3 )}$ & $\mathbf{( 2 1 , 7 )}$ \\
Depressão intermitente & $\mathbf{5}$ & $(4,3)$ \\
Mania & 1 & $(4,3)$ \\
Ciclotimia & 1 & $(13,0)$ \\
Psicose esquizoafetiva & 3 & $(13,0)$ \\
Distúrbio do pânico & 3 & $(8,7)$ \\
Distúrbio de ansiedade generalizada & 2 & $(13,0)$ \\
Fobias & 3 & $(8,7)$ \\
Alcoolismo & 2 & $(8,7)$ \\
Traços personalidade anormal & 2 & $(13,0)$ \\
Personalidade lábil & 3 & $(4,3)$ \\
Personalidade anti-social & 1 & $(13,0)$ \\
Tentativa de suicídio & 3 & \\
\hline
\end{tabular}


Tabela 4. Sintomas identificados pelo "syndrome check-list" (n=38).

\begin{tabular}{lc}
\hline Syndrome check-list (PSE) & $n(\%)$ \\
\hline SD (depressão simples) & $23(60,5)$ \\
IC (perda de interesse e concentração) & $23(60,5)$ \\
WO (preocupação) & $22(57,9)$ \\
OD (outros sintomas depressivos) & $19(50,0)$ \\
TE (tensão) & $18(47,4)$ \\
IT (irritabilidade) & $17(44,7)$ \\
ED (características depressivas especiais) & $17(44,7)$ \\
LE (perda de energia) & $15(39,5)$ \\
\hline
\end{tabular}

Tabela 5. Diagnósticos segundo as classes do PSE $(n=38)$.

\begin{tabular}{|c|c|c|}
\hline Classes do PSE & & $n(\%)$ \\
\hline Depressão inibida & $\mathbf{R}+$ & $10(26,3)$ \\
\hline Depressão neurótica & $\mathbf{N}+/ \mathbf{N} ?$ & $8(21,2)$ \\
\hline Psicose depressiva & D+ & $2(5,3)$ \\
\hline Psicose esquizofrênica & $\mathrm{S}+/ \mathrm{S} ?$ & $4(10,5)$ \\
\hline $\begin{array}{l}\text { Mania e estados } \\
\text { afetivos mistos }\end{array}$ & $\mathrm{M}+$ & $1(2,6)$ \\
\hline Sintomas inespecíficos & $\mathrm{X}$ & $6(15,8)$ \\
\hline Exame normal & $\mathrm{NO}$ & $7(18,4)$ \\
\hline
\end{tabular}

Os sub-escores do PSE sugeriram que as síndromes inespecíficas sejam a principal tendência sintomatológica dos pacientes da amostra (mais de $75 \%$ da amostra), em detrimento das manifestações psicóticas, que foram observadas em menos de $25 \%$ dos casos. Sintomas depressivos (Tabela 4) foram os achados mais frequentes da "syndrome checklist" do PSE. Vinte pacientes (9 homens e 11 mulheres; 52,6\%) encontravam-se deprimidos no momento avaliação (Tabela 5). Desses, 11 tinham também antecedentes depressivos segundo SADS-L e 4 apresentaram depressão no passado, mas estavam atualmente assintomáticos. Dezesseis pacientes preencheram critérios diagnósticos do DSM-III-R para transtorno depressivo orgânico $(k=0,4)$, o que conferiu confiabilidade adicional para os diagnósticos estabelecidos pelo PSE.

Testes estatísticos não-paramétricos foram utilizados para comparar os grupos de pacientes deprimidos e não-deprimidos (Qui-quadrado de Pearson para tabelas 2x2, ou teste exato de Fisher, quando diante de limitações numéricas para o uso do primeiro). Estimativas de risco relativo ( $R R \mathrm{e}$ $O R$ ) com $95 \%$ de intervalo de confiança foram utilizadas para se testar associações entre variáveis psiquiátricas e clínicas, com o intuito de se verificar eventuais fatores de risco para morbidade depressiva. Os dois grupos não diferiam significantemente quanto às variáveis demográficas, incluindo idade ( $F=1,29 \mathrm{NS})$, sexo ( $t=1,59 \mathrm{NS})$, cor $(t=0,91 \mathrm{NS})$, estado civil ( $t=3,22 \mathrm{NS})$, nível de instrução $(t=0,15 \mathrm{NS})$, ou tempo de doença $(t=0,66 \mathrm{NS})$. Não houve associação entre uso de esteróides e depressão $(\mathrm{P}=1,000)$. Contudo, a ocorrência de depressão segundo PSE correlacionou-se positivamente com atividade da doença $\left(\chi^{2}=4,062, \mathrm{df}=1, \mathrm{P}=0,04 ; O R=4,667,95 \% \mathrm{CI}_{\mathrm{OR}}=0,995\right.$ $21,895)$ e com a ocorrência de hipertensão intracraniana ( $\mathrm{P}=0,11, R R=1,813,95 \% \mathrm{CI}_{\mathrm{RR}}=1,306$ 2,516). Não foram encontradas associações entre depressão e presença de epilepsia ( $\mathrm{P}=0,629)$, lesões em espaço liquórico $(\mathrm{P}=0,621)$ ou gravidade do déficit cognitivo $(\mathrm{P}=0,569)$. História pregressa de transtornos depressivos correlacionou-se fortemente com a ocorrência de depressão atual $\left(\chi^{2}=7,620\right.$, $\left.\mathrm{df}=1, \mathrm{P}=0,006 ; R R=2,139,95 \% \mathrm{CI}_{\mathrm{RR}}=1,268-3,607 ; O R=14,667,95 \% \mathrm{CI}_{\mathrm{OR}}=1,590-135,322\right)$.

\section{DISCUSSÃO}

As estimativas de prevalência de alterações mentais na NCC são altamente variáveis, dependendo da origem das amostras de pacientes estudadas e da metodologia utilizada para o diagnóstico psiquiátrico. Em serviços de neurologia, alterações psíquicas são descritas com frequência em associação a síndromes hipertensivas e epilépticas, enquanto formas psíquicas puras seriam menos comuns ${ }^{31,32}$. Porém, nesses estudos, raramente os distúrbios mentais são definidos com exatidão, por não serem utilizados instrumentos apropriados para o diagnóstico psiquiátrico. Em função disso, os casos que cursam com 
perturbações menos intensas passam provavelmente desapercebidos. Por outro lado, a maioria dos estudos feitos por psiquiatras, tanto no começo do século como recentemente ${ }^{9}$, estimaram em até 75$100 \%$ o índice de pacientes acometidos por transtornos mentais na NCC.

O presente estudo inova e contribui em relação aos anteriores, ao procurar determinar a ocorrência de transtornos mentais através de instrumentos apropriados para o diagnóstico psiquiátrico e em uma amostra de pacientes oriundos de um serviço de neurologia, identificando nela uma alta prevalência de alterações depressivas.

Não é possível, a partir deste estudo, estabelecer estimativas da prevalência de depressão na NCC como um todo. Não foram utilizados grupos-controle para outras doenças crônicas nãoneurológicas (como, por exemplo, doenças sistêmicas e doenças que não acometem o SNC), pois este cuidado metodológico estava além dos objetivos iniciais do estudo. Portanto, não se podem excluir fatores independentes da NCC que respondam por parte da morbidade psiquiátrica encontrada. Além disso há, na comunidade, um número desconhecido de indivíduos com NCC que não se utilizam de ambulatórios especializados (possivelmente por terem doença mais branda), além de casos assintomáticos. Possivelmente, esses indivíduos apresentam menor grau de acometimento psiquiátrico. Como não dispomos de informações confiáveis sobre a prevalência real da NCC em nosso meio, ficam limitadas as generalizações desses achados.

Chama a atenção a alta taxa de pacientes que receberam o diagnóstico de transtornos depressivos $(52,6 \%)$ e a ocorrência menos frequente de distúrbios psicóticos. A ausência de predominância do sexo feminino entre pacientes deprimidos é, segundo alguns autores, forte evidência a favor de distúrbio do humor secundário a causas orgânicas ${ }^{33}$. Alguns índices de sofrimento cerebral, como a presença de hipertensão intracraniana e de sinais de atividade da doença, associaram-se positivamente com a ocorrência de depressão, nos pacientes da amostra, achados que têm alguma implicação etiológica. A HIC é a síndrome neurológica que mais frequentemente determina manifestações psíquicas na $\mathrm{NCC}^{31,32}$. Quanto à atividade da doença cerebral, na NCC, nenhum estudo até o momento tentou correlacioná-la com a psicopatologia. Embora inédita, é uma constatação coerente: doença em atividade significa presença de reações inflamatórias e quebra de $\mathrm{BHE}^{17}$. Em outras doenças que acometem o SNC, como o lupus eritematoso sistêmico e a esclerose múltipla, sinais de atividade inflamatória e imunológica também correlacionaram positivamente com a ocorrência de alterações do humor ${ }^{34,35}$. Na NCC parenquimatosa, a sintomatologia neurológica reflete atividade inflamatória do hospedeiro ${ }^{36}$; perturbações psíquicas também foram observadas na evolução do tratamento da NCC com drogas parasiticidas, fase em que o processo inflamatório é especialmente importante ${ }^{22}$. O presente estudo incluiu doentes acometidos por lesões correspondentes a diferentes estágios evolutivos dos cisticercos, sem delimitar regiões cerebrais de maior interesse. Estudos prospectivos com amostras mais homogêneas, ou com pacientes selecionados segundo localização e atividade das lesões, são necessários para a validação dos achados.

$\mathrm{Na}$ amostra estudada, entre todas as variáveis testadas, aquela com a qual se deu a associação mais significante com a ocorrência de depressão, foi a de antecedentes pessoais de distúrbios do humor $(\mathrm{p}=0,006)$. Esse achado é coerente com a vasta literatura dos transtornos mentais orgânicos ${ }^{37,38}$ : história de depressão anterior ao início do quadro neurológico, ao lado de antecedentes familiares de doença afetiva são fatores de risco relevantes para o desenvolvimento de depressão nos pacientes após acidente vascular cerebral ${ }^{39}$ ou com esclerose múltipla ${ }^{33}$. A presença desses antecedentes significaria maior vulnerabilidade biológica para o desencadeamento de depressão, servindo a agressão orgânica como evento precipitador. Na amostra, não foi possível identificar associação entre depressão e presença de antecedentes familiares de transtornos do humor. Contudo, foi pequeno o número de parentes de primeiro grau dos pacientes com NCC que tinham indícios de doença mental (4 casos), sendo ainda necessário ressaltar que esse levantamento não foi feito através de instrumento padronizado. 


\section{CONCLUSÕES}

1. É frequente o acometimento psiquiátrico e neuropsicológico entre os pacientes ambulatoriais portadores de NCC.

2. Diferentemente do que se tem registrado na literatura, estes pacientes tendem a apresentar mais transtornos afetivos do que quadros psicóticos, sendo a inespecificidade dos sintomas a principal tendência psicopatológica.

3. Entre os pacientes que receberam diagnóstico de depressão, a ausência de predomínio do sexo feminino é uma possível evidência de etiologia orgânica.

4. Atividade da doença neurológica e ocorrência de hipertensão intracraniana podem ser fatores de risco para o aumento da morbidade psiquiátrica nesses pacientes, sendo necessários estudos adicionais.

5. História psiquiátrica prévia foi, para os pacientes da amostra, o principal fator de risco para a ocorrência de depressão, sendo esse achado congruente com a literatura sobre outros transtornos mentais orgânicos.

\section{REFERÊNCIAS}

1. Del Brutto OH, Sotelo J. Neurocysticercosis: an update. Rev Infect Dis 1988;10:1075-1087.

2. Canelas HM. Neurocisticercose: incidência, diagnóstico e formas clínicas. Arq Neuropsiquiatr 1962; 20:1-16.

3. Del Brutto OH, Noboa CA. Late-onset epilepsy in Ecuador: aetiology and clinical features in 225 patients. J Trop Geogr Neurol 1991;1:31-34.

4. Flisser A. Neurocysticercosis in Mexico. Parasitol Today 1988;4:131-137.

5. Gutierrez EJS, Ospina IG. La taeniasis y cysticercosis en Mexico. Salud Publ Mex 1986;28:556.

6. Lombardo L, Mateos JH, Estañol B. La cisticercosis cerebral en México. Gac Med Mex 1982;118:1-16.

7. Loo L, Braude A. Cerebral cysticercosis in San Diego. Medicine 1982;61:341-359.

8. Scharf D. Neurocysticercosis: two hundred thirty-eight cases from a California hospital. Arch Neurol 1988;45:777-780.

9. Tavares AR Jr. Aspectos neuropsiquiátricos da neurocisticercose humana. Tese de Doutorado, Escola Paulista de Medicina. São Paulo, 1994.

10. Centers for Disease Control and Prevention. Neurocysticercosis: update. International Task Force for Disease Erradication. MMWR 1992;41:691-698.

11. McCormick GF, Zee C, Heiden J. Cysticercosis cerebri: review of 127 cases. Arch Neurol 1982;39:534-539.

12. Grisolia JS, Wiederholt WC. CNS cysticercosis. Arch Neurol 1982;39:540-544.

13. Brown WJ, Voge M. Cysticercosis: a modern day plague. Pediatr Clin North Am 1985;32:953-969.

14. Pawlowsky ZS. Cestodiasis: taeniasis, diphyllobothriasis, hymenolepiasis and others. In Warren KS, Mahmoud AAF (eds). Tropical and geographical medicine. New York: McGraw-Hill, 1984:471-486.

15. Schantz PM, Sarti-Gutierrez E. Diagnostic methods and epidemiologic surveillance of Taenia solium infection. Acta Leidensia 1989;57:153-163.

16. Flisser A, Pérez-Montfort R, Larralde C. The immunology of human and animal cysticercosis: a review. Bull World Health Organ 1979;57:839-856.

17. Sotelo J, Guerrero V, Rubio F. Neurocysticercosis: a new classification based on active and inactive forms. Arch Intern Med 1985; 145:442-445.

18. Sotelo J, Torres B, Rubio-Donnadieu F, Escobedo F, Rodríguez-Carbajal J. Praziquantel in the treatment of neurocysticercosis: long term follow-up. Neurology 1985;35:752-755.

19. Tavares AR Jr. Psychiatric disorders in neurocysticercosis (Letter). Br J Psychiatry 1993;163:839.

20. Livramento JA. Síndrome do líquido cefalorraqueano na neurocisticercose: estudo crítico sobre a evolução da imunidade humoral. Tese de Docência Livre, Faculdade de Medicina da Universidade de São Paulo. São Paulo, 1986.

21. Livramento JA, Costa JM, Machado LR, Nóbrega JPS, Spina-França A. ELISA (IgG e IgM) no LCR e soro na neurocisticercose em tratamento com praziquantel. Arq Neuropsiquiatr 1985,43:1967-1974.

22. Takayanagui OM. Neurocisticercose: avaliação da terapêutica com praziquantel. Arq Neuropsiquiatr 1990;48:11-15.

23. Machado LR, Nóbrega JPS, Barros NG, Livramento JÁ, Bacheschi LA, Spina-França A. Computed tomography in neurocysticercosis: a 10-year long evolution analysis of 100 patients with na appraisal of a new classification. Arq Neuropsiquiatr 1990;48:414-418.

24. Suh DC, Chang KH, Han MH, Lee SR, Han MC, Kim CW. Unusual MR manifestations of neurocysticercosis. Neuroradiology 1989;31:396-402.

25. Spitzer RL, Endicott J. Schedule for affective disorders and schizophrenia (life-time) - SADS-L. 3.Ed. NIMH: Clinical Research Branch Collaborative Program on the Psychobiology of Depression, May 1978-Sept1979.

26. Wing JK, Cooper JE, Sartorius N. The measurement and classification of psychiatric symptoms: an instruction manual for the present state examination and CATEGO program. Cambridge: Cambridge Univ Press, 1974. 
27. American Psychiatric Association. Diagnostic and statistical manual of mental disorders. 4.Ed. Washington DC: American Psychiatric Association, 1994.

28. Folstein MF, Folstein SE, Mchugh PRH. Mini-Mental State: a practical method for grading the cognitive state of patients for the clinician. J Psychiatr Res 1975;12:198.

29. Bertolucci PHF, Brucki SMD, Campacci SR, Juliano Y. O mini-exame do estado mental em uma população geral: impacto da escolaridade. Arq Neuropsiquiatr 1994;52:1-7.

30. Strub R, Black FW. Mental status examination in neurology, 2.Ed. Philadelphia: FA Davis, 1986.

31. Canelas HM. Cisticercose do sistema nervoso central. Revista de Medicina 1963;47:75-89.

32. Lima JGC. Cisticercose encefálica: aspectos clínicos. Tese de Docência Livre, Escola Paulista de Medicina. São Paulo, 1966.

33. Popkin MK, Tucker GJ. "Secondary" and drug-induced mood, anxiety, psychotic, catatonic, and personality disorders: a review of the literature. J Neuropsychiatr Clin Neurosci 1992;4:369-385.

34. Miguel EC, Pereira RMR, Pereira CAB, et al. Psychiatric manifestations of systemic lupus erythematosus: clinical features, symptoms, and signs of central nervous system activity in 43 patients. Medicine 1994;73:224-232.

35. Moller A, Wiedemann G, Rohde U, Backmund H, Sonntag A. Correlates of cognitive impairment and depressive mood disorders in multiple sclerosis. Acta Psychiatr Scand 1994;89:117-121.

36. Shandera WX, White AC Jr, Chen JC, Diaz P, Armstrong R. Neurocysticercosis in Houston, Texas: a report of 112 cases. Medicine 1994;73:37-52.

37. Feinstein A, Ron MA Psychosis associated with demonstrable brain disease. Psychol Med 1990, 20:793-803.

38. Forlenza OV, Vieira AHG Filho, Nóbrega JPS, et al. Psychiatric manifestations of neurocysticercosis: a study of 38 patients from a neurology clinic in Brazil. J Neurol Neurosurg Psychiatry 1997;62:612-616.

39. Brumback RA. Is depression a neurologic disease? Behavioral Neurol 1993;11:79-104. 